\title{
Analysis of a Prescription Drug Prior Authorization Program in a Medicaid Health Maintenance Organization
}

KENNETH T. LAPENSEE, MPH, PhD

\begin{abstract}
OBJECTIVE: To determine the factors important in approving prescription reimbursement under prior authorization $(\mathrm{PA})$ in a Medicaid managed care organization (MCO).

METHODS: A cross-sectional statistical analysis was performed using administrative data for one month of PA requests to an MCO with more than 250,000 Medicaid recipients in the northeast United States.

RESULTS: More than $95 \%$ of PA reviews resulted in payment for the originally prescribed products. The most common treatments involved were atypical antipsychotics, antacids, antidepressants, antihypertensives, anticonvulsants, and Cox-2 inhibitors. The rejection rate for nonformulary products was $7.1 \%$ while that for formulary products was $3.7 \%$. Nevertheless, most drugs requiring PA were formulary-listed, with protocols to reinforce prescription guidelines. Rejection of reimbursement was inversely related to patient age. Most likely to be authorized were drugs for smoking cessation, pain, and nausea, while those least likely to be approved were multivitamins, sleep aids, and high-cost antidepressants.

CONCLUSION: Although nonformulary products are more frequently subject to PA, $78.6 \%$ of PA procedures are performed in response to requests for formulary-listed products. The PA rejection rate for this Medicaid MCO was small; $4.4 \%$ overall and $7.1 \%$ for nonformulary versus $3.7 \%$ for formulary drugs.
\end{abstract}

KEYWORDS: Pharmaceuticals, Prior authorization, Managed care, Medicaid, Reimbursement, Formulary

J Managed Care Pharm. 2003(9)1: 36-44
Authors

KENNETH T. LAPENSEE, MPH, PhD, is Senior Vice President, Managed Care, Cambridge Pharma Consultancy, a unit of IMS Health, Inc. Hampton, New Jersey.

AUTHOR CORRESPONDENCE: Kenneth T. LaPensee, MPH, PhD, Senior Vice President, Managed Care, Cambridge Pharma Consultancy, 80 Broad St., 35th Floor, New York, NY 10004. Tel: (646) 274-1800 and (908) 537-6462; Fax: (630)604-8191; E-mail: Ken_LaPensee@cambridge-pharma.com

Copyright $\odot$ 2003, Academy of Managed Care Pharmacy. All rights reserved.
$\mathrm{T}$ he purpose of this study was to determine whether and under what circumstances prior authorization (PA), a labor-intensive administrative procedure, results in approval of the PA. The specific research questions are: (a) How frequently does the PA procedure result in denying reimbursement for a prescribed drug, and (b) What are the factors that predict reimbursement denial in a PA intervention program?

Health plans and health policy analysts are interested in whether or not PA programs affect the original prescription rate and the actual utilization rate of affected drugs, given the expensive and administratively intensive nature of these procedures. The null hypothesis is that PA of drugs does not significantly affect the utilization rate of prescribed pharmaceuticals. Second, the vast majority of PAs are approved, regardless of therapeutic class or patient characteristic. A corollary to the null hypothesis is that no particular drug class or patient age and gender has any relationship to the outcome of PA.

Even if the null hypothesis is true, a PA program may give rise to a "sentinel effect" in preventing prescribing of drugs on the list of PA drugs. This effect could be well worth the cost of the administration of the program, but this question is beyond the scope of the present project and has been documented elsewhere.

\section{Background}

\section{The Prior Authorization Procedure for Pharmaceuticals}

Since no two drugs are exactly alike in their efficacy and safety for particular patients, health plans with formularies use management tools such as PA to influence prescribing for certain drugs. In this procedure, physicians call the health plan to obtain authorization to prescribe a drug on the PA list for a particular patient because the prescriber believes that the drug is the most appropriate treatment for that patient. Health plan staff typically review patient characteristics, diagnosis, and prescription history and adjudicate the physician's request. A question of major importance is whether this procedure really changes the ultimate outcome in terms of the patient receiving the originally prescribed medication. A secondary question is whether there is a difference between formulary-listed drugs and nonformulary drugs in the frequency with which PA is used and in the frequency with which this procedure actually reverses prescription choice by denying reimbursement on the basis of formulary status.

In 1997, 87\% of all managed care organizations (MCOs) used PA. ${ }^{2}$ By 1999 , this had increased to $88.3 \%$. A breakdown of the use and results of PA by MCOs of various types is shown in Table $1 .{ }^{3}$

According to the 2000 Novartis Pharmacy Benefit Report, ${ }^{3}$ plans with closed formularies, such as the extant 
Analysis of a Prescription Drug Prior Authorization Program in a Medicaid Health Maintenance Organization

TABLE 1 Use of PA by Health Plan Lines of Business

\begin{tabular}{lccccc} 
& $\begin{array}{c}\text { Commercial/ } \\
\text { Group }\end{array}$ & Medicaid & Medicare & Overall & Research Site HMO \\
\hline Use PA & $94.1 \%$ & $82.9 \%$ & $81.4 \%$ & $88.3 \%$ & Yes \\
\hline $\begin{array}{l}\text { Apply to select } \\
\text { therapeutic classes }\end{array}$ & $79.8 \%$ & $67.9 \%$ & $81.8 \%$ & $77.9 \%$ & All classes \\
$\begin{array}{l}\text { Average number PAs } \\
\text { requested PMPM }\end{array}$ & 0.08 & 0.03 & 0.14 & 0.08 & 0.09 \\
\begin{tabular}{l} 
Average \% of approvals \\
\hline Sources for national figures: Novartis Pharmacy Benefit Report.
\end{tabular} & $74.0 \%$ & $69.0 \%$ & $74.0 \%$ & $95.6 \%$ \\
\hline
\end{tabular}

research site health maintenance organization (HMO), a Medicaid MCO, report the highest number of PAs and the lowest percentage of approvals. In this regard, the extant research site HMO data from January 2001, analyzed in this report, appear to be in line with expectations in terms of the number of PAs but far above national figures in terms of the numbers of requests that are approved. As noted below, figures for the research site HMO reflect the final rejection rate after any appeals.

A pharmacy benefit manager owned by the PacifiCare Health Systems reported to a congressional subcommittee $e^{4}$ that their data (which were not provided to the committee) showed that PAs for nonformulary products occurred in only $1 \%$ of claims, and $75 \%$ of these requests were approved. One may reasonably speculate that this low request rate for nonformulary products may illustrate a sentinel effect ${ }^{1}$ in which physicians were "alerted" to either the formulary status of drugs in local health plans by their patients through health plan communication or through prior experience with PA denials. For example, IMS Health, a national vendor of prescription data, offers a proprietary service to pharmaceutical firms $s^{5}$ based on the precept that managed care formularies exert an influence in local markets beyond their membership where they have a significant share of membership because doctors become used to prescribing according to their formularies. Off-formulary prescribing for members that comprise a significant portion of physician practices results in frequent patient complaints about lack of reimbursement, which usually leads to the prescribing of an alternative drug with better coverage under the patient's plan or PA procedures. ${ }^{6}$ As mentioned below, health plans rely on the sentinel effect to reduce nonformulary prescribing to a point where PA approval rates are high but nonformulary claim volume is low. The latter consideration needs to be figured into costbenefit calculations for PA justification.

\section{Managed Care Organization Formularies and Prior Authorization of Pharmaceuticals}

In recent years, pharmaceutical costs have outstripped most other aspects of health care in cost growth. MCOs have primarily used 2 tools in their efforts to control pharmaceutical budg- et growth: formularies and PA, which tend to work synergistically. The effect of PA in controlling utilization, the focus of this research, needs to be understood in the context of the health plan's formulary.

The PA of pharmaceuticals by health plans is controversial. Health plans maintain that PA helps to curb inappropriate drug use through its sentinel effects. Critics answer that it raises administrative costs for all affected parties. Health plans report costs of $\$ 10$ to $\$ 25$ per authorization request while more than $80 \%$ of the requests are approved. ${ }^{2}$ Some doctors see PA as threatening to their independence and authority in diagnosis and treatment. Some patients may consider PA to be interference with their right to receive the best quality medical care. Pharmaceutical companies consider PA an unnecessary barrier to access to their products. Pending legislation in various states could influence procedures to overturn PA denials, the length of time in which a coverage decision must be rendered, disclosure of denials to patients and doctors, and many other rules that will drive up the cost of the PA procedure. It is reasonable to question whether cost savings from the procedure offset the administrative expense and whether drug PA in a litigious environment invites avoidable complaints and lawsuits by interfering with the practice of medicine by denying prescription reimbursement.

In spite of these doubts and pitfalls, the use of PA appears to be growing, attendant to the increased use of drug formularies by employers and health plans. In 2000, nearly all HMOs and the vast majority of preferred-provider organizations (PPOs) used drug formularies. ${ }^{7}$ This observation is consistent with personal communications from various sources, including executives at CIGNA HealthCare, with a large commercial population, who claim that PA has restrained per-member-per-month (PMPM) costs for Cox-2 inhibitors (rofecoxib and celecoxib) in CIGNA programs that include PA. ${ }^{8}$

\section{Effect of a Closed Formulary in a Commercial HMO}

In a recent study, Motheral et al. reported on the utilization and financial impact of a commercial HMO's closed formulary. ${ }^{9}$ In this retrospective cohort study with a pre-post design, formulary cases (patients) had a higher generic drug refill rate, lower total 


\begin{tabular}{lccc}
\hline TABLE 2 & Frequencies of Variables & \\
\hline Variable & Value & Frequency & Percentage \\
\hline Formulary status & Yes & 17,308 & $78.6 \%$ \\
& No & 4,701 & $21.4 \%$ \\
\hline Reimbursement & Paid & 20,888 & $94.9 \%$ \\
status & Adjusted & 147 & $0.7 \%$ \\
& Rejected & 974 & $4.4 \%$ \\
\hline Patient age & Children (0 to 17) & 2,581 & $12.4 \%$ \\
& Young adults (18 to 34) & 2,870 & $13.7 \%$ \\
& Early middle age (35 to 50) & 6,687 & $32.0 \%$ \\
& Later middle age (50 to 64) & 5,215 & $25.0 \%$ \\
& Elderly (65+) & 3,527 & $16.9 \%$ \\
\hline Gender & Male & 9,023 & $41.0 \%$ \\
& Female & 12,986 & $59.0 \%$ \\
\hline
\end{tabular}

claims, and lower mean brand claims in the postformulary period than matched controls, controlling for age, sex, chronic disease score, and utilization in the preformulary period.

One of the study-dependent variables was the mean number of PAs per subject, defined as the total number of PAs divided by the number of months of eligibility during the research period. With the implementation of the formulary, the closed formulary group had a greater increase in the mean number of PAs per patient-month $(P<0.0001)$. Not surprisingly, PAs tend to be more frequent under closed formularies. Because PA and formulary coverage can affect commercial HMO enrollment decisions, as noted above, but not Medicaid program enrollment, the effects of the application of the PA procedure in commercial and Medicaid programs may not be completely comparable.

\section{Consumer Perceptions of Prior Authorization Programs}

Another recent study by Momami et al. explored consumer perceptions of 4 drug management strategies, including PA. ${ }^{10}$ This study used a cross-sectional mail survey design that targeted MCO enrollees residing in the mid-Atlantic region. Reporting on the results, the authors state:

"...[R]espondents mildly agreed that PA limits their access to the best medications.... They felt neutral that this policy results in less-effective medications, compromises the quality of their drugs, and affects their compliance with their drugs. Finally, respondents mildly disagreed that PA makes it more convenient to get the prescribed drugs. Respondents' overall attitude toward PA was somewhat negative."

Furthermore, the authors reported that PA was one of the factors that motivated members to join or leave an $\mathrm{HMO}$, and members of more liberally managed plans such as PPOs were more likely to ask their pharmacists questions about PA.

These data suggests that many patients find the PA procedure at least moderately annoying and potentially a factor in member dissatisfaction and plan disenrollment. However, it was not viewed as negatively in this particular study as the imposition of a formulary itself, which of course often results in increased PA. As noted above, consumer perceptions of PA could effect enrollment in a commercial plan but not a Medicaid managed care program, and this could be a consideration in how the procedure is applied in the 2 types of programs.

\section{Cost-Benefit Analyses of Prior Authorization}

Some cost-benefit analyses of the PA procedure have recently been conducted. In one health plan that included both commercial and Medicaid members, the average administrative cost of a limited PA program covering bupropion (standard release formulation), the Cox-2 drugs, the glitazone antidiabetic drugs, antifungals, zafirlukast/montelukast, and sildenifil, was \$17.87 per PA at a volume of 936 requests per month. ${ }^{11}$ No cost savings for the Medicaid population were reported, although cost savings for the Cox-2 drugs, the glitazones, and sildenifil were reported for the commercial population. This study did not take into account any factor for a supposed sentinel effect. The administrative costs included employee salaries and indirect administrative costs. The savings calculation used in the study was: savings in drug spend $=(\#$ denied $\mathrm{x} \$ \mathrm{Rx})-(\$$ substitute $\mathrm{Rx})$. The net savings to the health plan $=$ (monthly PAs $\mathrm{x}$ administrative \$) - reduction in drug spend.

A recent study reported that step therapy for Cox-2 inhibitors was cost effective. ${ }^{12}$ Step therapy requiring treatment failure due to gastrointestinal discomfort is the protocol commonly incorporated into health plan PA procedures for Cox-2 drugs. The study was reanalyzed because cost data for the products in the study (celecoxib, rofecoxib, naproxen sodium, and nabumetone) was inaccurate. The reanalysis confirmed that step therapy might be cost effective, but that a 3-tier copay plan in which the Cox-2 drugs are assigned to the third tier is more cost effective considering plan cost and member choice.

An international meta-analysis of studies of the impact on health care quality and cost of restrictive formularies ${ }^{13}$ was unable to draw definitive conclusions but suggested that PA may be effective in controlling drug costs without increasing costs in other areas of medical expenditures. A study to determine whether PA of topical tretinoin for acne is in the best interest of health insurers and, if so, to determine the optimal member age for topical tretinoin PA, indicated that PA for topical tretinoin is of no great benefit to insurers. ${ }^{14}$ The authors conclude that although they generally support the use of PA, elimination of prior authorization altogether for this condition treatment would result in at most a 12\% loss, about one penny PMPM, that would be balanced by reduced inconvenience for patients and reduced expenditure of time for doctors.

A study in the hospital setting ${ }^{15}$ of the PA of antimicrobial use determined that requiring preapproval for selected parenteral agents can decrease antimicrobial expenditures and improve susceptibilities to antibiotics without compromising patient outcomes or length of hospital stay. While the circum- 


\section{Analysis of a Prescription Drug Prior Authorization Program in a Medicaid Health Maintenance Organization}

stances of this type of PA are very different from PA in the ambulatory setting, the findings of this study may have implications for PA of antibiotics in the ambulatory setting where considerations of antibiotic resistance and response to antimicrobial therapy can be somewhat similar.

A 1995 study described an evaluation of Medicaid HMO PMPM costs, preauthorization processes, drug utilization, and provider and member educational efforts. After one year of managing the pharmaceutical benefit of a former Medicaid population, plan PMPM pharmacy costs decreased, PA request and call activity decreased, and drug market shares shifted toward formulary agents. ${ }^{16}$ Provider and member acceptance of the restrictive formulary grew as consistent interaction with the $\mathrm{HMO}$ increased HMO staff familiarity with specific patient cases, and providers grew more familiar with the HMO's criteria for decisions regarding PR.

Another 1995 study ${ }^{17}$ concluded that PA requirements in a Medicaid population might be highly cost effective with regard to expenditures for NSAIDs, drugs that have very similar efficacy and safety but have substantial variation in cost.

\section{The Research Site HMO Drug Formulary}

The extant research site HMO uses a "closed" formulary that covers only certain brand drugs. Furthermore, some of the covered formulary drugs require PA for reimbursement, a practice common in other managed care plans. The PA procedure as described below is used by the health plan to screen prescriptions for medical necessity according to utilization guidelines that have been incorporated into the PA protocol and procedure.

\section{The Research Site Formulary and Prior Authorization Process}

The research site drug formulary has a closed design and was developed to cover medically necessary and cost-effective prescription products for self-administration in the ambulatory setting.

The goal of the HMO's PA process is to ensure that medication regimens that are high cost, high risk, or with narrow therapeutic indices are used appropriately in the care of members. The PA process is required for

- limited-use agents such as orphan drugs (payment for orphan drugs will be based on the "Office of Orphan Product Development" guidelines for medical necessity);

- all brand-name medications when there is an A-rated generic equivalent available, except as noted in the generic medications section;

- all nonformulary medications (for example, sildenafil, simvastatin, topiramate);

- medications and regimens under concurrent clinical review, regimens that are outside the parameters of use approved by the U.S. Food and Drug Administration (FDA) or accepted standards of care, for example, a longer than 14-day course of antibiotics;

- prescriptions that exceed $\$ 500$;

- injectable medications other than insulin, epinephrine, and

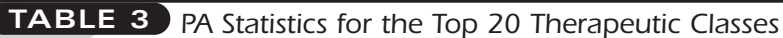

\begin{tabular}{|c|c|c|}
\hline Drug Class & $\begin{array}{l}\text { Number PA } \\
\text { Procedures }\end{array}$ & Percentage of PA \\
\hline Atypical antipsychotics & 2,696 & $12.2 \%$ \\
\hline Antacids & 1,916 & $8.7 \%$ \\
\hline Antidepressants & 1,830 & $8.3 \%$ \\
\hline Antihypertensives & 1,172 & $5.3 \%$ \\
\hline Anticonvulsants & 1,067 & $4.8 \%$ \\
\hline Cox-2 inhibitors & 825 & $3.7 \%$ \\
\hline Hypnotics & 802 & $3.6 \%$ \\
\hline Nonsedating antihistamines & 788 & $3.6 \%$ \\
\hline Male sexual disorder & 713 & $3.2 \%$ \\
\hline Analgesics and narcotics & 693 & $3.1 \%$ \\
\hline Antifungals & 599 & $2.7 \%$ \\
\hline ACE Inhibitors & 506 & $2.3 \%$ \\
\hline Cholesterol-lowering & 479 & $2.2 \%$ \\
\hline HmG-CoA inhaled asthma steroids & 460 & $2.1 \%$ \\
\hline Analgesics and NSAIDs & 439 & $2.0 \%$ \\
\hline Oral hypoglycemics & 403 & $1.8 \%$ \\
\hline Antidepressant 5HT reuptake & 294 & $1.3 \%$ \\
\hline Reverse nucleoside for AIDS & 250 & $1.1 \%$ \\
\hline Electrolytes & 248 & $1.1 \%$ \\
\hline CNS stimulants & 214 & $1.0 \%$ \\
\hline
\end{tabular}

vitamin B-12 and injectables administered by physicians or other skilled professionals (certain injectables are available exclusively through the injectable program);

- all prescriptions that exceed plan limits, for example, prescriptions for the antiasthmatic drug montelukast in doses greater than the maximum dose of one tablet per day; and

- prescriptions processed by nonnetwork pharmacies.

Upon receiving a PA request, the research site HMO will ensure that the recipient "continues a course of treatment without interruption by (a) authorizing continued treatment from the current prescriber or (b) facilitation of an uninterrupted transition to an equivalent course of treatment received from providers within the HMO network." Protocols must comply with FDA-approved guidelines and not contribute to a pattern of fraud or abuse.

Physician and pharmacy providers receive regular communications detailing changes in the PA process. Doctors have the primary responsibility for obtaining PA of medications. When possible, the prescriber obtains PA before the member goes to the pharmacy. If a nonnetwork doctor writes a prescription and that prescriber refuses to contact the health plan for authorization, then it is the responsibility of the primary care physician to obtain authorization. 
Analysis of a Prescription Drug Prior Authorization Program in a Medicaid Health Maintenance Organization

(TABLE 4$)$ Frequency of Requests for Individual Drugs (Frequency $>1.0 \%$ of PAs)

\begin{tabular}{|c|c|c|c|c|c|c|}
\hline \multirow{2}{*}{$\begin{array}{l}\text { Prescription Ranked } \\
\text { by PA Frequency }\end{array}$} & \multirow{2}{*}{$\begin{array}{l}\text { Formulary } \\
\text { Drug }\end{array}$} & \multirow{2}{*}{$\begin{array}{l}\text { Number of } \\
\text { PA Requests }\end{array}$} & \multirow{2}{*}{$\begin{array}{c}\text { Percent } \\
\text { of Requests }\end{array}$} & \multicolumn{2}{|c|}{ Disposition } & \multirow[b]{2}{*}{ Percent Rejected } \\
\hline & & & & Accepted & Rejected & \\
\hline Lansoprazole & Yes & 1,350 & $6.1 \%$ & 1,301 & 49 & $3.6 \%$ \\
\hline Olanzapine & Yes & 1,280 & $5.8 \%$ & 1,242 & 38 & $3.0 \%$ \\
\hline Risperidone & Yes & 940 & $4.3 \%$ & 912 & 28 & $3.0 \%$ \\
\hline Bupropion SR & No & 586 & $2.7 \%$ & 533 & 53 & $11.1 \%$ \\
\hline Metoprolol XL & No & 545 & $2.5 \%$ & 509 & 36 & $6.6 \%$ \\
\hline Generic temazepam & Yes & 449 & $2.0 \%$ & 444 & 5 & $1.1 \%$ \\
\hline Topiramate & No & 379 & $1.7 \%$ & 349 & 30 & $7.9 \%$ \\
\hline Rofecoxib & Yes & 287 & $1.3 \%$ & 277 & 10 & $3.5 \%$ \\
\hline Nephrocaps & Yes & 239 & $1.1 \%$ & 234 & 5 & $2.1 \%$ \\
\hline Atorvastatin & Yes & 230 & $1.0 \%$ & 225 & 5 & $2.2 \%$ \\
\hline Zolpidem tartrate & Yes & 228 & $1.0 \%$ & 224 & 4 & $1.8 \%$ \\
\hline Fluoxetine & Yes & 225 & $1.0 \%$ & 222 & 3 & $1.3 \%$ \\
\hline Sertraline & Yes & 220 & $1.0 \%$ & 212 & 8 & $3.6 \%$ \\
\hline Tramadol & Yes & 212 & $1.0 \%$ & 205 & 7 & $3.3 \%$ \\
\hline Paroxetine & Yes & 212 & $1.0 \%$ & 205 & 7 & $3.3 \%$ \\
\hline Celecoxib & Yes & 211 & $1.0 \%$ & 202 & 9 & $4.3 \%$ \\
\hline
\end{tabular}

\section{Prior Authorization Process for Physicians}

To obtain a PA, the physician or his or her staff contacts the research site HMO. Requests should be reviewed within 24 hours of receipt. If the request fails to meet the research site HMO-approved criteria, a medical director reviews the request, relying upon accepted clinical and state guidelines. Arrangements are made for the patient to continue on therapy until the request is resolved.

Appeals are reviewed by the medical director's office. Prescribers should receive a written notice of the decision within 72 hours of receipt of the appeal. The physician, patient, and pharmacy should all be notified of the medical director's decision on the PA within 72 hours.

\section{Methods}

\section{Analytical Technique}

This study used a cross-sectional statistical analysis of health plan administrative data for one month of PAs submitted to the research site HMO by physicians. These data consisted of 22,009 PA records for the month of January 2001, the first month for which these data elements had been recorded for the health plan. Although this was the first month of recording, the PA program had been in existence at the research site HMO for more than 5 years. Thus, these data do not represent new or extraordinary circumstances for the program, although seasonality of claims may be a significant limitation of the study.

Data analysis consisted of calculating univariate frequencies, bivariate cross tabulations, and the calculation of a binary logistic regression model of the likelihood that a request for PA would be denied, given drug types and patient demographics. The binary multiple logistic regression technique was chosen for the main data analysis because it helps to identify which fac- tors independently predict reimbursement denial under PA.

Administrative data were used in this project because they contain information about every request for PA. These data are limited since the diagnosis and reasons for the request are not recorded. The therapeutic class of the product requested was used as a proxy for the diagnosis.

\section{Results}

The majority (78.6\%) of the drugs subject to PA are formulary-listed products. PA is thus used far more often to validate appropriate use of listed drugs for which the health plan likely receives financial discounts than it is used to review the use of nonformulary products. Still, the number of nonformulary reviews is substantial, with nearly 5,000 such reviews in one month.

Demographically, a majority (59\%) of the patients who were subject to PA for prescription drugs were female, consistent with Medicaid enrollment demographics; $57 \%$ of the patients were between ages 35 and 65 years, and 17\% were age 65 or older (Table 2).

\section{Reimbursement Outcomes}

The overwhelming majority (more than 95\%) of PA reviews resulted in the payment of claims for the originally prescribed product. One flaw in these data is that there is no indication of whether the claim went through an appeals process prior to being paid. The PA process adjusted a small number of claims. An adjusted transaction occurs when a PA has been approved but the patient does not pick up the drug at the pharmacy. In this situation, the transaction is "reversed" (not "rejected") to adjust for the amount paid. However, in this paper, the calculation of the rejection rate includes consideration of reversed claims in the denominator: we consider them to be authorized and paid because that was the intention of the plan. 
Only $4.4 \%$ of the requests were finally rejected. In one month, this amounted to fewer than 1,000 requests. If January is a typical month for the program, then in one year, about 10,000 to 12,000 requests will be rejected.

\section{Therapeutic Classes}

Twenty therapeutic classes accounted for $1 \%$ or more, each, of the PAs (Table 3). The top 6 therapeutic classes were atypical antipsychotics, antacids, antidepressants, antihypertensives, anticonvulsants, and the Cox-2 specific inhibitors. This list of drug classes mirrors the experience of many state Medicaid programs that have PA programs for pharmaceuticals. ${ }^{18}$ The top 10 (of 83) therapeutic classes accounted for $56.5 \%$ of all PAs, and the top 20 accounted for $74.1 \%$ of all requests. Most of these therapeutic classes were represented in the PA file by only 3 or fewer drugs.

\section{Individual Drugs}

The great majority of PAs among the most frequently represented therapeutic classes discussed above were for branded pharmaceutical products (Table 4). Of 87 most requested products, accounting for nearly $64 \%$ of all requests, 70 were for branded pharmaceuticals ( $55.3 \%$ of all requests), 7 were for vitamins and minerals (4.8\% of all requests), and 10 were for generic products (3.7\% of all requests). The frequency of generics and vitamins requested increases when products of lower frequency are considered, but, overall, the majority of PAs are made for branded pharmaceuticals. Thus, one of the primary outcomes, and indeed an implicit function of the research site HMO's PA program, is to monitor and control the utilization of branded pharmaceuticals.

Since the research site $\mathrm{HMO}$ is a Medicaid managed care program, this roster of products reflects a mix that characterizes the treatment of Medicaid populations. For example, the high frequency of atypical antipsychotic drugs such as ziprasidone, risperidone, and quentiapine reflects the significance of Medicaid in the coverage of pharmacologic treatment of psychosis. However, other products listed seem fairly typical of those that might be subject to PA in a commercial HMO covering the general population. For example, heartburn medications such as lansoprazole and omeprazole and antidepressants such as fluoxetine, citalopram, and sertraline are among the most commonly used drugs in any health insurance plan. ${ }^{19}$

Since vitamins and minerals are generally not covered by health plans unless they are prescribed by a physician and are considered medically necessary, their coverage is always subject to PA. Given the predominance of PA for a limited number of branded products and the evident purpose of this administrative procedure to control the utilization of branded pharmaceuticals, the following presentation of results focuses on the PA process applied to the branded products listed in Table 4.

\section{Bivariate Results: Prior Authorization and Formulary Status}

It was noted earlier that PA is required for all nonformulary drugs and also to ensure the medical necessity for and proper utilization of certain formulary drugs. An example of the latter case is rofecoxib, a formulary Cox-2 inhibitor. A patient must first fail on 3 traditional NSAIDs before a claim for rofecoxib is accepted and reimbursable to the pharmacy.

One of the central questions of this research is whether the PA approval of formulary-listed products is proportionately more likely than that of nonformulary products. Although the vast majority of PAs of both formulary-listed and nonformulary products were approved, significantly more PA requests for nonformulary products were rejected than should be expected by chance alone $(P<.0001)$. This seems reasonable because although the PA procedure is used to ensure appropriate medical utilization of formulary-listed drugs, PA for nonformulary drugs involves the additional step of first checking to see whether there is a "therapeutically equivalent" branded or generic formulary-listed drug, and then checking whether the patient has already failed to respond to the identified "equivalent" formulary-listed product. We were unable to obtain data regarding the percentage of treatments with branded formulary products that have failed, so we cannot determine the proportion of formulary-listed products that have turned out not to be truly equivalent to nonformulary products in the cases of specific patients.

This result appears to support the argument that PA is one of the tools by which the MCO enforces the use of brand formulary products since the probability of rejection of the prescription is higher when a nonformulary brand product is prescribed. Using a nonformulary product requires an extra burden of support for approval-not only must it be therapeutically appropriate, the same criterion as that for a formulary drug requiring PA, but the nonformulary drug must also be therapeutically superior to the brand formulary drug, for the specific patient. Showing superiority of the brand nonformulary product would require demonstration that the patient failed treatment on the formulary product.

\section{Particular Drugs and Prior Authorization Rejection}

The top 10 drugs in terms of reversed prescriptions include lansoprazole, olanzapine, topiramate, clotrimazole-betamethasone diproprionate, rofecoxib, quinapril hydrochloride tablets, celecoxib, sertraline, oxycodone-acetaminophen tablets, and citalopram. Although the numbers of rejections are small for all of the individual drugs and only 2 of the top 10 drugs were nonformulary products, the nonformulary products had considerably higher rejection rates than the formulary products. This is in line with the overall results showing that the rejection rate for all nonformulary products was $7.1 \%$ while that for formulary products was $3.7 \%$.

The most common reason for reversing the prescription of formulary drugs was the application of quantity limitations per month. These limits are in addition to the general plan limits in which a "maximum of a 34-day supply or 150 units (whichever is less) of medication is eligible for coverage." The plan limits statement reads, "Prescribed medications of regimens that are for nonformulary drugs or over 34-days supply/150 units require PA." 


\section{Prescription Rejection by Patient Demographics}

Age: The likelihood that a product will fail to be reimbursed under PA at this Medicaid HMO is inversely related to age, with the widest divergence in actual versus expected rejections in the youngest age group ( 0 to 17 years) and the oldest age group (65+ years). There is no hypothesis for this association at present, and its use in this study is simply as a covariate, controlled in the multivariate analysis.

Finally, the PA requirements on the products might be more likely to be met for the older patient. This association will be assessed again when multivariate analysis results are reported. (The age categories were 0 to 17,18 to 34,35 to 49,50 to 64 , and $65+$ years [Medicare/Medicaid dually eligible patients]).

Gender: There does not appear to be any association between gender and the likelihood of having a prescription reversed under PA, and we know of no hypothesis that posits such an association.

\section{Prescription Rejection by Therapeutic Category}

Prescriptions from particular therapeutic categories are more likely to be reversed under PA. The top 10 categories in terms of impact on prescribing under the PA program are angina drugs, multivitamins and mineral formulations, sleep aids, antismoking drugs, antifungals, ACE inhibitors, anticonvulsants, anxiolytics, A-listed antiemetics, and nonsedating antihistamines. For most of these therapeutic classes, one or two drugs account for nearly all of the PAs as well as the rejections.

\section{Multivariate Analysis Procedure}

In order to determine which factors in the PA data independently determine the outcome of a PA, a binary multiple logistic regression analysis using the dependent variable "Rejected" was employed, signifying the rejection of approval for a particular request. Rejected is a categorical dummy-coded variable for which a case is scored 1 if reimbursement authorization is denied and 0 if the request is either adjusted or paid.

Based on the bivariate cross-tabulation results above, the therapeutic class dummy variables were entered in a preliminary model along with age group and formulary status. Of these, anxiolytics and the drug classes containing furosemide and multivitamin soft gels were discarded as not statistically significant in the preliminary modeling.

The remaining variables mentioned above were entered into the first block of a 2-block regression model. A second block of variables representing individual, commonly prescribed drugs was entered into the regression model. This was done to determine whether individual drugs added additional information about the results of PA over and above that provided by therapeutic classes. Only 2 of these drugs, the analgesic/narcotic (oxycondone/acetaminophen) and the drug for smoking cessation, depression, and anxiety (bupropion), added additional statistically significant information about the outcomes of PAs.

\section{Model Results}

The binary logistic regression results suggest that, for the most part, the covariates are not intercorrelated. The highest correlation was between the request for PA of angina drugs and age group of the patient. In line with the low intercorrelations, the results of multivariate analysis were similar in direction to the bivariate relationships discussed earlier.

Using the prior probabilities of group membership (Rejected $[1,0]$ ) of about $0.05,0.95$ in the analysis maximized the probability of correct case classification by the binary logistic regression procedure, but the classification power of the model was modest, at about $68 \%$ correct. The results of the HosmerLemeshow goodness-of-fit test were not statistically significant. This finding implies that the model variables and, by extension, the data elements collected by the research site HMO are not highly predictive of the results of a PA. It is possible that an analysis of the specific reasons why the PA of products results in approval or rejection (eg., exceeding plan limits) would be more powerful in helping to prospectively classify the results of PA. However, these reasons are not recorded in the PA claims data system at present.

The prescription of multivitamins, sleep aids, and the psychotropic drug bupropion (prescribed for smoking cessation) resulted in significantly higher odds of having a PA rejected. The prescription of angina, smoking cessation (other than bupropion), antifungals, ACE inhibitors, antiemetics, formulary drugs, in general, and the pain drug oxycondone/acetaminophen resulted in significantly lower odds of having a PA rejected. Drugs for smoking cessation and drugs to control pain and nausea were the most likely to be approved by PA. Greater patient age was associated with significantly lower odds of having a PA rejected.

\section{Discussion}

The finding that the formulary status of a prescribed drug influences the outcome of PA, with formulary drugs more likely to be authorized, was anticipated and is consistent with the work of others cited above (Motheral et al., 2000; Momami et al., 2000).

The association of age group with approval of PA was not anticipated and has not been reported in the literature. This association of PA approval with older patient age is apparently over and above that which is due to the tendency of older people to use any particular class of drugs that were retained in the model. At present, there is not a hypothesis regarding this association.

The relative ease of getting approval for smoking-cessation drugs at the research site HMO appears to be part of a general emphasis at the health plan on primary prevention of smokingrelated disorders. In view of the recent settlements between the tobacco industry and state Medicaid programs, this emphasis may reflect a broader policy of encouraging smoking cessation within Medicaid programs. ${ }^{20}$

The relative ease of getting approval for pain and nausea drugs would understandably be associated with a general policy within 
managed care of increasing quality of life for patients with serious illness such as cancer and, perhaps, AIDS.

On the other hand, multivitamins are not generally covered by health plans, with only special exceptions, and the tendency of a PA program to reject requests for reimbursement of these products should be expected. The only basis for approving such a request would be in the rare case of malnutrition, extremely poor diet, or some sort of physiological abnormality such as enzyme deficiency.

The prescription of sleep aids causes a review of patient diagnosis and raises the question of why over-the-counter products cannot be used. Admittedly, this is a very poorly understood medical area, and much more precision in the targeting of sleep products and the diagnostic criteria for prescribing them is needed.

Bupropion sustained-release is the first representative of a new class of psychotropic drugs called the serotonin-norepinephrine reuptake inhibitors. This drug commands a price premium over the more established selective serotonin reuptake inhibitors such as fluoxetine and citalopram. Health plan officials report that it is often prescribed as a smoking cessation aid. In this application, the drug is subject to PA.

More than 95\% of PA reviews in the study sample resulted in payment for the originally prescribed products. The most common treatments affected by the PA process were atypical antipsychotics, antacids, antidepressants, antihypertensives, anticonvulsants, and Cox-2 inhibitors. The rejection rate for nonformulary products $(7.1 \%)$ was nearly double that for nonformulary products (3.7\%). Nevertheless, most drugs requiring PA were formularylisted, but with protocols to reinforce prescription guidelines related to standards of appropriate utilization.

Our model variables and, by extension, the data elements collected by the research site $\mathrm{HMO}$, are not highly predictive of the results of a PA. It is possible that an analysis of the specific reasons why the PA of products results in approval or rejection (eg., exceeding plan limits) would be more powerful in helping to prospectively classify the results of PA. These reasons are not recorded in the PA claims data system at present, but it seems that there would be value in recording them to better determine why physicians prescribe nonformulary drugs and why they prescribe in ways that are at variance with PA criteria. In fact, this could be a basis for the review of PA criteria in terms of their conformity with local medical practice.

The administration of the research site HMO PA program requires considerable resources. The pharmacy director has devoted considerable time to the creation of the program and monitors its continuing development. Two full-time RNs adjudicate the PA claims, which number between 70 and 100 per day. Many of these claims are easily adjudicated because the PA criteria are simple, although some go through an appeals process that involves interacting with physicians. These RNs are also involved in helping to set PA policy for particular drugs and ongoing research to maintain the currency of PA criteria. A programmer/analyst devoted about one-half time to the PA claims data system provides computer programming and statistical analysis for routine and special reports of PA claims. An administrative assistant devotes approximately one-quarter time to supporting this RN team for PA administration.

Drug PA programs reflect the desire of payers to reduce prescription drug costs and channel utilization toward formularylisted drugs but also often represent an attempt to reinforce health plan or community standards of medical practice and treatment guidelines to encourage appropriate utilization. For example, a course of antibiotics longer than 2 weeks is more than health plan guidelines allow and would be unusual in terms of recommended utilization. Another example would be that biologics for rheumatoid arthritis would not generally be prescribed until a patient had failed to respond to methotrexate. Although nonformulary products are more subject to PA more frequently, by proportion, the majority of PA procedures are performed in response to requests for formulary-listed products. In a commercial health plan, many products for which the health plan receives a rebate may be prior-authorized for conformance to plan guidelines. A major unanswered question is the extent to which PA protocols truly reflect the standard of care in medical practice and significant public health concerns. There is a need to verify that the clinical basis for the PA criteria are grounded in sound, evidence-based medicine.

The predominant use of PA for nonformulary drug prescribing could support the assertion by Medicaid programs that while PA may have some financial motivation, its basic rationale is the medically appropriate use of pharmaceutical products. This question is all the more significant in view of the Pharmaceutical Manufacturers Association (PhRMA) lawsuit against the state of Maine regarding the imposition of PA under the state's Medicaid program for drugs not listed on the state's formulary. PhRMA contended that the PA program was financially motivated and not in the interest of beneficiaries. In March 2001 and again in June 2001, the U.S. Circuit Court of Appeals found that "although PA is triggered by a manufacturer's refusal to participate in the Maine prescription program, testimony from the court record indicates that the final decision to require PA for a particular drug is based primarily on clinical criteria applied by health care professionals." ${ }^{21}$ Specifically, placement of a drug on the PA list may only be decided by the state's Medicaid Drug Utilization Review Committee, composed of physicians and pharmacists licensed in Maine. PhRMA is appealing this decision to the U.S. Supreme Court.

PA seems likely to remain a widely used tool for Medicaid and non-Medicaid managed care programs because its wider use suggests that health plan sponsors believe that drug-benefit PA reduces costs. ${ }^{20}$ However, the ultimate fate of PA in commercial health insurance in pharmacy benefit programs will probably not be decided through litigation regarding interference in medical practice but by the growing emphasis on consumer cost sharing in recent health insurance benefit design, exemplified by tiered formularies. Some health plans contend that if patients are required to pay extra for particular drugs, they should not be 


\section{Analysis of a Prescription Drug Prior Authorization Program in a Medicaid Health Maintenance Organization}

forced to undergo restrictive administrative procedures to be reimbursed. On the other hand, PA will probably continue to find use in reinforcing standards of care. If the trend toward greater consumer cost sharing continues, PA will probably become unnecessary as a way to increase the utilization of formulary products since plans will rely on patient out-of-pocket cost sensitivity to channel utilization toward preferred products.

\section{Limitations}

Population: The research site HMO is a Medicaid MCO. Its covered population is, therefore, discontinuously eligible for benefits as people lose and gain income or assets. On the other hand, if a state has a Medicaid managed care program under a Section 1115 waiver from the U.S. Department of Health and Human Services, a beneficiary has no other coverage option. This could make it somewhat different from private HMOs that cover commercial populations since enrollment patterns in a commercial HMO generally depend on the stability of their employer customers and on employee health plan choices when employers offer multiple options. In a commercial HMO, employees sometimes have the option of joining another health plan during an open-enrollment period or even opting out of the employer plan in favor of participation in a spouse's health plan. An employer might change health plans based on complaints about a drug formulary, or a beneficiary might change health plan options. Thus, a drug formulary might have implications for enrollment and disenrollment, if, for example, a patient's preferred drug is not covered under a closed formulary. In considering the results of the present study, we must keep in mind that the differences between commercial and Medicaid populations might limit the generalizability of our conclusions.

Sampling: For administrative reasons, we were only able to access one month of claims data in a timely fashion. Since a Medicaid population may be seasonal in that beneficiaries may be better able to find employment at some times during the year than others, and many illnesses are seasonal, the limitations of a single month's data are significant and can limit the generalizability of our conclusions.

\section{Conclusion}

In this 250,000-member Medicaid HMO, 78.6\% of drug PA procedures in January 2001 were performed in response to requests for formulary-listed products. The PA rejection rate for this Medicaid MCO was small: 4.4\% overall and 7.1\% for nonformulary versus $3.7 \%$ for formulary drugs. The generalizability of these conclusions may be limited because a Medicaid population has significant differences from commercially insured populations in terms of socioeconomic status and enrollment choice.

\section{ACKNOWLEDGMENT}

The author gratefully acknowledges the research guidance and help with the manuscript provided by Dona Schneider, PhD, his professor and academic advisor at the New Jersey School of Public Health.

\section{DISCLOSURES}

No outside funding supported this study. All of the research reported in this article was financed by author Kenneth $\mathrm{T}$. LaPensee as part of pursuing a master's degree in public health at the New Jersey School of Public Health. His tuition for that degree program was also self-financed except for 6 credit hours at the beginning of the program that were paid for with funds from an internship grant by the U.S. Public Health Service; the grant expired several years before the time the manuscript was prepared. Consulting project work provided to the pharmaceutical and biotechnology industries funds his salary at his current employer, Cambridge Pharma Consultancy.

\section{REFERENCES}

1. Bazelon Center. Medicaid formulary policies: access to high-cost mental health medications. November 1999. Available at: http://www.bazelon.org/formulary.html.

2. Novartis Pharmacy Benefit Report 1998. Novartis Pharmaceutical Corp., Inc., Parsippany, NJ.

3. Novartis Pharmacy Benefit Report 2000. Novartis Pharmaceutical Corp., Inc., Parsippany, NJ.

4. Prescription Solutions, Inc. Testimony provided to the House Energy and Commerce/Health Subcommittee, February 15, 2001. The Green Sheet. February 2001:26:3.

5. IMS Health Inc. Managed Care Strategic Summary Report (proprietary) January 2002.

6. Yuan Y, Duckwitz N. Doctors and DTC. Pharm Executive. August 2002:7.

7. Aventis Managed Care HMO/PPO Digest 2001. Aventis Pharmaceuticals, Bridgewater, NJ.

8. In a conversation with Daniel M. Cave, Vice President, Managed Pharmacy Operations, CIGNA HealthCare (March 2001).

9. Motheral B, Delate T, Shaw J, Henderson R. The effect of a closed formula ry in the face of real-life enrollment and disenrollment patterns. J Managed Care Pharm. 2000;6(4):293-97.

10. Momami A, Odedina F, Rosenbluth S, Madhavan S. Drug-management strategies: consumer perspectives. J Managed Care Pharm. 2000;6(2):122-28.

11. Mascari TA, Johnson KA. Cost of administering a prior-authorization program at a health plan, and associated cost savings. Poster presented at: AMCP Conference. J Managed Care Pharm. 2000;6(5):376.

12. Tucker G, Moore A, Avant D, et al. A cost analysis of four benefit strategies for managing a Cox-II inhibitor. J Managed Care Pharm. 2001;7(3):224-27.

13. Lexchin J. Effects of restrictive formularies in the ambulatory care setting. Am J Managed Care. 2002;8(1):69-76.

14. Feldman SR, Fleischer AB Jr., Chen GJ. Is PA of topical tretinoin for acne cost effective? Am J Managed Care. 1999;5(4):457-63.

15. White AC Jr., Atmar RL, Wilson J, Cate TR, Stager CE, Greenberg SB. Effects of requiring PA for selected antimicrobials: expenditures, susceptibilities, and clinical outcomes. Clin Infect Dis. 1997;25(2):230-39.

16. Smith WW, Perry SC. Implementation of a pharmacy benefit to a managed Medicaid population. Med Interface. 1995;8(12):84-87,97.

17. Smalley WE, Griffin MR, Fought RL, Sullivan L, Ray WA. Effect of a prior-authorization requirement on the use of nonsteroidal anti-inflammatory drugs by Medicaid patients. N Engl J Med. 1995;332(24):1612-17.

18. In a conversation with Martha McNeill, Director of Product Management, Texas Department of Health, Bureau of Vendor Drugs Director of Pharmaceutical Benefits, Texas State Medicaid Program (April 2000). 19. Novartis Pharmacy Benefit Report 2000, Novartis Pharmaceutical Corp., Inc., Parsippany, NJ.

20. In a conversation with the Pharmacy Director of the Research Site HMO (name withheld due to confidentiality agreement with the HMO) (October 2001).

21. Henneberry, J. Addendum to State Pharmaceutical Assistance Programs: The Maine Rx Program. Available at: http://www.nga.org. 